\section{Brain tuberculoma in a non-endemic area}

\author{
Robert M. Lober, Anand Veeravagu, \\ Harminder Singh
}

Department of Neurosurgery, Stanford Hospitals and Clinics, Stanford, CA, USA

\section{Abstract}

Brain tuberculoma has previously accounted for up to a third of new intracranial lesions in areas endemic with tuberculosis, but is unexpected in the United States and other Western countries with improved disease control. Here we show the importance of considering this diagnosis in at-risk patients, even with no definitive pulmonary involvement. We describe a young man who presented with partial seizures and underwent craniotomy for resection of a frontoparietal tuberculoma. He subsequently completed six months of antituberculosis therapy and was doing well without neurological sequelae or evidence of recurrence five months after completion of therapy. With resurgence of tuberculosis cases in the United States and other Western countries, intracerebral tuberculoma should remain a diagnostic consideration in at-risk patients with new space occupying lesions. Mass lesions causing neurological sequelae can be safely addressed surgically and followed with antituberculosis therapy.

\section{Introduction}

Brain tuberculoma has previously accounted for up to a third of new intracranial lesions in areas endemic with tuberculosis, ${ }^{1}$ but is unexpected in the United States and other Western countries with improved control of systemic disease. Here we show the importance of considering this diagnosis in at-risk patients, even with no definitive pulmonary involvement.

\section{Case Report}

A 24 year-old man who had immigrated from Ethiopia six years prior presented to our Emergency Department with intermittent left leg shaking for two days. His left leg felt tired and he was reluctant to stand on it. He was previously healthy and had no history of fever, chills, night sweats, or weight loss. He was well appearing with no abnormal findings on physical and neurological examination. A computed tomography (CT) scan showed right fron- toparietal edema. A contrast-enhanced scan was performed, revealing an associated frontoparietal enhancing mass. magnetic resonance imaging (MRI) of the brain demonstrated a $2.6 \times 1.6 \mathrm{~cm}$ mass with surrounding edema (Figure 1). CT of chest, abdomen, and pelvis revealed periceliac and peiraortic lymphadenopathy, and questionable but no definitive pulmonary nodules. Having received the Bacillus Calmette-Guérin vaccine, his tuberculin sensitivity test was positive. He was started on levetiracetam for treatment of simple partial seizures, and dexamethasone for treatment of vasogenic edema. He underwent right parietal craniotomy with intraoperative monitoring for resection of the lesion and tissue diagnosis. The underlying dura was soft and uninvolved. Opening the dura revealed a superficial mass with well defined margins that easily peeled away from the underlying parenchyma (Figure 2). Pathological examination demonstrated necrotizing granulomas with peripheral palisading epithelioid histiocytes, giant cells, and acid fast bacilli, consistent with cerebral tuberculoma (Figure 3). Blood cultures and HIV testing were negative. Following surgery, he had no neurological deficits and had no further seizure activity. He was rapidly tapered from steroids and continued on levetiracetam for an additional month. He completed a six-month regimen of isoniazid, rifampin, ethambutol, and pyrizinamide, and was doing well without neurological sequelae five months after completion of therapy.

\section{Discussion}

The tuberculoma is a parenchymal reaction to hematogenous seeding of the brain surface, with its indolent course differing from and only occasionally associated with tubercular abscesses or meningitis. ${ }^{2,3}$ It may arise from the conglomeration of several so-called miliary tubercles, i.e., incipient tuberculomas, around the outer sheaths of small cerebral blood vessels, with eventual central caseation and fibrous encapsulation. The larger masses may be more prone to central caseation, leading to purulent transformation and abscess formation. There may be vasculitis, giant cell systems and outward extension with plaque formation against the meninges, which can become inflamed but are otherwise relatively impervious.

Geographic and temporal patterns of brain tuberculomas have followed the dynamic trends of the systemic disease, influenced by socioeconomic development, public health measures and, in recent decades, association with HIV infection. ${ }^{4,5}$ In the United States and other Western countries with improved control of tuberculosis, these cases are understandably rare and unexpected, potentially leading to
Correspondence: Robert M. Lober, Stanford Hospitals and Clinics, Department of Neurosurgery, 300 Pasteur Drive, R200/MC:5826, Stanford, CA 94305, USA.

Tel. +1.650.7250701 - Fax: +1.650.7237918.

E-mail: roblober@stanford.edu

Key words: tuberculoma, tuberculosis, anti-tuberculous therapy.

Conflict of interests: the authors declare no potential conflict of interests.

Received for publication: 5 February 2012.

Revision received: 4 April 2012.

Accepted for publication: 5 April 2012.

This work is licensed under a Creative Commons Attribution NonCommercial 3.0 License (CC BYNC 3.0).

(C) Copyright R.M. Lober et al., 2013

Licensee PAGEPress, Italy

Infectious Disease Reports 2013; 5:e1

doi:10.4081/idr.2013.e1

delayed diagnosis and treatment. ${ }^{6}$ In nonendemic areas the majority of central nervous system tuberculosis involves adult immigrants from areas of high prevalence, ${ }^{7}$ as was exemplified by the patient in the current report. Nonimmigrant cases generally involve immunosuppressed adults, most often due to HIV, but also associated with alcoholism, diabetes mellitus, malignancy, or use of steroids or agents blocking tumor necrosis factor activity.

Several very thorough descriptions of large series of brain tuberculomas have come from authors practicing in areas endemic to the disease in the last century. ${ }^{1,6,8}$ In India in the 1950 's and 1960's, tuberculomas accounted for almost one third of space occupying lesions in the brain, comparable to the number of newly diagnosed gliomas. ${ }^{1}$ The majority of our understanding of the disease process comes from reports such as these, since during the same period one Western author noted only three cases at a British neurosurgical service that had treated 2200 intracranial tumors. ${ }^{9}$ Since that era there have been decreasing numbers of reports, as a PubMed search revealed only five non-overlapping large case series with at least 50 patients in the contemporary English medical literature, i.e., the last five decades. ${ }^{1,2,10,11}$

Without concomitant meningitis, brain tuberculomas closely resemble neoplasms, causing seizures, progressive focal neurological deficits, and occasionally refractory edema or increased intracranial hypertension. ${ }^{1,6,8}$ There are no clinical features distinct from other space occupying lesions. ${ }^{7}$ The differential diagnosis among these patients is that of almost any ring-enhancing lesion, generally indistinguish- 
able from other equally plausible causes within endemic and non-endemic areas, such as lymphoma, glioma, bacterial or fungal abscess, neurocysticercosis, or toxoplasmosis. ${ }^{7}$ Given the lack of systemic evidence for active tuberculosis, we had favored the diagnosis of glioma in this patient preoperatively.

The majority of tuberculomas are isodense to brain parenchyma on CT scan, with variable calcification. ${ }^{11}$ MRI characteristics are diverse and dependent upon the amount of caseation, but most T1-weighted images show an isointense core with a hypointense rim, and most T2weighted images show a hypointense core with a hyperintense rim, which may be dificult to distinguish from some stages of cerebritis or cerebral abscess, including tubercular abscess.

These lesions commonly occur in the cerebral or cerebellar hemispheres, with a greater predilection for the infratentorial compartment. ${ }^{12}$ In this case the dura was not grossly involved despite the superficial location, but there are reports describing meningeal nodules and unusual sites such as within the ventricles..$^{13}$ Concomitant tuberculous meningitis may be associated with contrast-enhancing basal exudates. ${ }^{7}$

Lumbar puncture often has a low diagnostic yield for tuberculoma and, given the significant perilesional edema and mass effect in the current patient, did not have a favorable riskbenefit ratio in this case. Previous authors have found that, although protein may be elevated, definitive acid-fast bacilli are inconsistently found in cerebrospinal fluid in cases of tuberculoma without meningitis. ${ }^{7}$ Similarly, lumbar puncture is often not useful for primary brain tumor, which was also a consideration preoperatively.

There is no established standard of surgical care for tuberculomas. Prior to effective antituberculous therapy, decompression alone without resection was recommended because of a high risk of resultant meningitis. In endemic locations where suspicion for tuberculoma is high with any newly discovered intracranial lesion, surgical excision or biopsy is usually reserved for when the diagnosis is in doubt or there is no response to empiric medical therapy for at least two months. ${ }^{14,15}$ However, surgery is always indicated in cases of intolerable intracranial hypertension.

Open or stereotactic biopsy is generally agreed upon as a minimum initial step in regions where such lesions are uncommon, ${ }^{16}$ but could still potentially be deferred in clear cases of reactivation of dormant tuberculosis. ${ }^{17}$ In cases of immunocompromise such as HIV, the wide differential diagnosis of enhancing brain lesions almost always necessitates brain biopsy for diagnosis. ${ }^{18}$ Given the low incidence and high association with HIV in the United States, only rarely will cases be diagnosed and treated preoperatively.
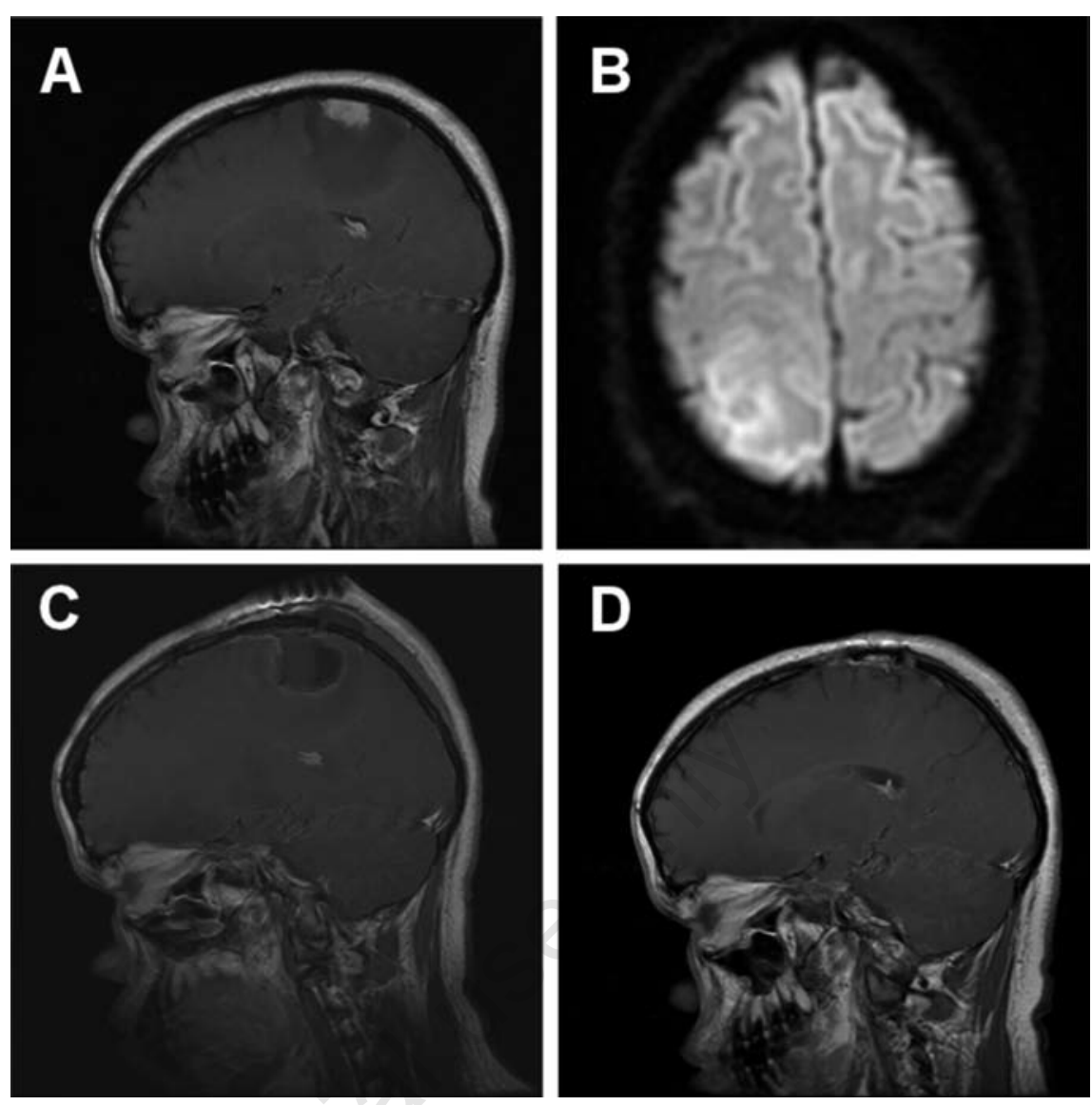

Figure 1. Sagittal T1-weighted magnetic resonance imaging (MRI) of the brain with gadolinium contrast. A) Preoperative image demonstrates a $2.6 \times 1.6 \mathrm{~cm}$ enhancing mass with surrounding edema. B) Axial diffusion-weighted MRI demonstrating perilesional edema but no solid areas of restricted diffusion to suggest abscess. C) Postoperative image one day after surgery demonstrating gross total resection. D) Postoperative image four months after surgery showing no recurrence.

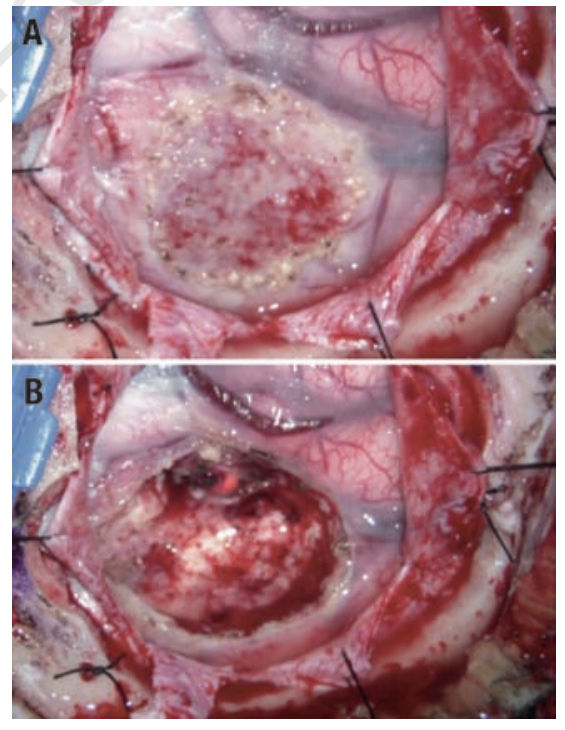

Figure 2. Intraoperative images of tumor. A) Superficial mass with well defined margins and surrounding normal brain tissue; B) resection cavity.
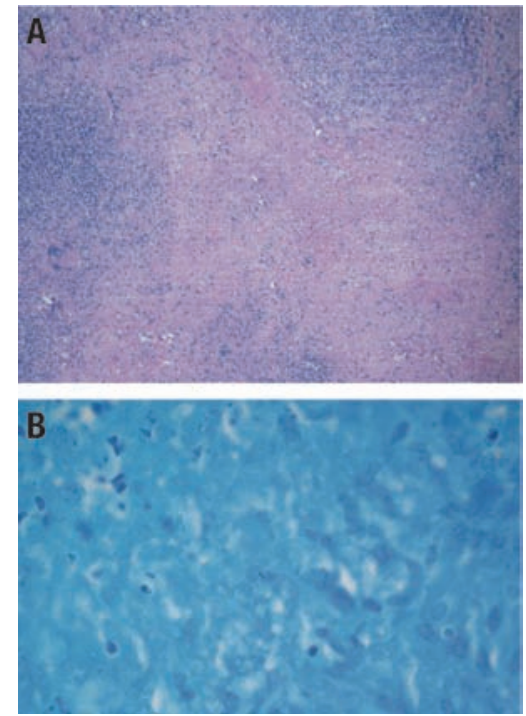

Figure 3. Pathological examination of tumor. A) Hematoxylin and eosin staining show necrotizing granulomas with peripheral palisading epithelioid histiocytes, giant cells. B) Acid fast bacillus. 
In the current case, preoperative imaging revealed a very superficial lesion that we surmised could be removed in its entirety with a small craniotomy, without any additional operative time or morbidity compared to that of open biopsy. A gross total resection was preferred in order to achieve greater survival benefit if pathological examination would have revealed malignant glioma.

When diagnosis certain, isoniazid, rifampin, and pyrazinamide penetrate cerebrospinal fluid and are considered the mainstay of treatment, while the benefits of less penetrating agents such as ethambutol and streptamycin are not proven. ${ }^{7}$ The optimum time for antibiotic therapy has not been clearly established. For tuberculous meningitis, six months of therapy is probably sufficient, although many authors recommend a year of treatment. For intracranial tuberculomas, recent recommendations on duration of therapy have come from expert opinion and small case series. One retrospective study of 18 patients in Saudia Arabia demonstrated radiographic resolution of all tuberculomas after one year of antituberculous therapy. ${ }^{15}$ Authors of a study of 17 patients in the United States with central nervous system tuberculosis, including nine tuberculomas, recommended prolonged anti-tuberculous therapy for 18 months. ${ }^{19}$

\section{Conclusions}

With resurgence of tuberculosis cases in the United States and other Western countries, intracerebral tuberculoma should remain a diagnostic consideration in at-risk patients with new space occupying lesions, even in the absence of definitive pulmonary involvement or cerebrospinal fluid evidence. Mass lesions causing neurological sequelae can be safely addressed surgically and followed with antituberculosis therapy.

\section{References}

1. Dastur HM, Desai AD. A comparative study of brain tuberculomas and gliomas based upon 107 case records of each. Brain 1965;88:375-96.

2. Sinh G, Pandya SK, Dastur DK. Pathogenesis of unusual intracranial tuberculomas and tuberculous space-occupying lesions. J Neurosurg 1968;29:149-59.

3. Whitener DR. Tuberculous brain abscess; report of a case and review of the literature. Arch Neurol 1978;5:148-55.

4. Corbett EL, Watt CJ, Walker N, et al. The growing burden of tuberculosis: global trends and interactions with the HIV epidemic. Arch Intern Med 2003;163:1009-21.

5. El Sahly HM, Teeter LD, Pan X, et al. Mortality associated with central nervous system tuberculosis. J Infection 2007;55: 502-9.

6. Bahemuka M, Murungi JH. Tuberculosis of the nervous system- a clinical, radiological and pathological study of 39 consecutive cases in Riyadh, Saudia Arabia. J Neurol Sci 1989;90;67-76.

7. Thwaites G, Fisher M, Hemingway C, et al. British Infection Society guidelines for the diagnosis and treatment of tuberculosis of the central nervous system in adults and children. J Infect 2009;59:167-87.

8. Arseni C. Two hundred and one cases of intracranial tuberculoma treated surgically. J Neurol Neurosurg Psychiatry 1958; 21:308-11.

9. Maurice-Williams RS. Tuberculomas of the brain in Britain. Postgrad Med J 1972;48:678-81.

10. Jinkins JR. Computed tomography of intracranial tuberculosis. Neuroradiol 1991; 33:126-35.

11. Wasay M, Kheeleani BA, Moolani MK, et al. Brain CT and MRI findings in 100 consecutive patients with intracranial tuberculoma. J Neuroimag 2003;13:240-7.

12. Dastur HM. Diagnosis and treatment of tuberculous disease of the CNS. Neurosurg Rev 1983;6:111-7.

13. Desai K, Nadkarnj T, Bhatjiwale M, Goel A. Intraventricular tuberculoma. Neurol Med Chir (Tokyo) 2002;42:501-3.

14. Al-Mefty 0 . Intracranial tuberculoma. J Neurosurg 1986;65:57.

15. Awada A, Daif AK, Pirani M, et al. Evolution of brain tuberculomas under standard antituberculous treatment. J Neurol Sci 1998;156:47-52.

16. Tandon PN. Brain biopsy in tuberculoma: the risks and benefits (letter). Neurosurg 1992;30:301.

17. Mayers MM, Kaufman DM, Miller MH. Recent cases of intracranial tuberculomas. Neurol 1978;28:256-60.

18. Bishburg E, Sunderam G, Reichman LB, Kapila R. Central nervous system tuberculosis with acquired immunodeficiency syndrome and its related complex. Ann Intern Med 1986;105:210-3.

19. Gropper MR, Schulder M, Sharan AD, Cho ES. Central nervous system tuberculosis; medical management and surgical indications. Surg Neurol 1995;44:378-85. 